\title{
Dheepan, un film postcolonial
}

\section{Anthony Goreau-Ponceaud and Paul Veyret}

\section{(2) OpenEdition}

Journals

\section{Electronic version}

URL: http://journals.openedition.org/echogeo/14456

DOI: 10.4000/echogeo.14456

ISSN: 1963-1197

\section{Publisher}

Pôle de recherche pour l'organisation et la diffusion de l'information géographique (CNRS UMR 8586)

\section{Electronic reference}

Anthony Goreau-Ponceaud and Paul Veyret, « Dheepan, un film postcolonial », EchoGéo [Online], Sur le Vif, Online since 15 February 2016, connection on 03 May 2019. URL : http://journals.openedition.org/ echogeo/14456; DOI : 10.4000/echogeo.14456

This text was automatically generated on 3 May 2019.

\section{(c) (i) (9)}

EchoGéo est mis à disposition selon les termes de la licence Creative Commons Attribution - Pas d'Utilisation Commerciale - Pas de Modification 4.0 International 


\title{
Dheepan, un film postcolonial
}

\author{
Anthony Goreau-Ponceaud and Paul Veyret
}

1 La lecture qui suit de Dheepan ${ }^{1}$ (2015) de Jacques Audiard est le fruit du croisement de deux regards : celui d'un spécialiste de la diaspora tamoule et celui d'un spécialiste du cinéma diasporique indien. Il nous est apparu que lors de sa remise de la Palme d'or au Festival de Cannes 2015, ce film avait été l'objet de nombreux malentendus, dont la cause était à la fois une méconnaissance du contexte politique du conflit sri-lankais et d'autre part une interprétation ne prenant pas suffisamment en compte la dimension postcoloniale du sujet. Dheepan est un film sur la diaspora tamoule, dans sa dimension humaine, et notre propos sera d'analyser le parcours d'un migrant dans un espace donné ; nous nous sommes interrogés également sur la façon dont Audiard place Dheepan dans une généalogie cinématographique hybride, à la fois issue du cinéma américain des années 60-70 et inscrite, par la nature de son propos, dans une problématique postcoloniale.

Dheepan, dans la continuité d'Un prophète (2009), dépeint la fascination de Jacques Audiard pour une forme de récit « d'intégration » qui tend plus vers le genre du film noir que vers celui de la comédie sociale et qui aboutit - dans le bruit et la fureur - à une forme d'aporie tant narrative que sociale. Le cinéaste, comme dans ses précédents films De battre mon cœur s'est arrêté (2005), De rouille et d'os (2012), est parti de l'idée de la représentation du «déphasage» (Rigoulet, 2015) d'un personnage par rapport à un milieu qui lui est radicalement étranger, comme par exemple le jeune Malik et les clans "ethniques » (Corses, Maghrébins...) de la Centrale dans le film Un prophète. Audiard place ce décalage créatif dans une matrice cinématographique qui croise ici le film noir et les récits de vétérans, le film de vigilante ${ }^{2}$ et l'épopée d'un survivant. Sur cette tradition cinématographique par nature hybride et aux frontières mouvantes, Audiard greffe un nouveau décalage, celui du diasporique ${ }^{3}$, dédoublant ainsi le prisme au travers duquel la société française est perçue. La banlieue française, objet qui sature les écrans de télévision et occupe une place marginale mais identifiable au sein du cinéma hexagonal, devient, pour Dheepan et son ersatz de famille, l'espace paradoxal d'accueil d'un migrant, vétéran perdu et anonyme de la guerre civile sri lankaise. En effet, le Pré Saint-Gervais, 
loin d'être le lieu idyllique et bucolique dont ils rêvent, un avant-goût de l'Eldorado britannique, est au contraire un espace de relégation sociale et identitaire.

\section{Dheepan, filiations cinématographiques}

3 Avec un héros fils caché d'Usbek des Lettres persannes devenu expert du cocktail molotov et d'un Travis Bickle tamoul roi de la machette et du gunfight, Dheepan a pu donner l'impression, en particulier à la critique française, de se perdre dans les approximations idéologiques d'un Escape from New York (New York, 1997) de Carpenter ou, pis encore, d'un Death Wish (Un Justicier dans la ville, 1974), avec son Charles Bronson exterminateur de petites frappes latinos. Bref, Audiard aurait mis un Tigre de l'Eelam tamoul ${ }^{5}$ dans sa banlieue pour mieux la nettoyer au kärcher ${ }^{6}$. Néanmoins, pour Audiard, le film de vigilante qui semblait se profiler au début de la rédaction du scénario n'était " pas intéressant » (Rigoulet, 2015) et il faut en effet tenter de voir Dheepan comme un objet hybride, un film postcolonial qui associe à l'univers déjà balisé de la banlieue française la question de la diaspora. Sur ce mode du décalage se trouvent ainsi superposés la question du rejet de la population d'origine immigrée française et le destin d'un membre de la diaspora tamoule : à la question de l'immigration s'agrège celle de la migration, et à la question de l'intégration des descendants des populations issues de l'ancien empire français se greffe celle de la trajectoire du diasporique.

4 Au renversement du point de vue déjà étalonné au cinéma dans des films comme Ma 6-T va crack-er (1997) de Jean-François Richet et La haine (1995) de Mathieu Kassovitz, qui privilégient le regard d'exclus sociaux qui miment des poses de gangstas américains, Audiard rajoute celui d'un autre exclu relégué dans les marges de l'histoire : celui d'un ancien combattant tamoul qui se retrouve par hasard au Pré Saint-Gervais (Seine-SaintDenis) et devient ainsi témoin privilégié et malgré lui de la transformation de la banlieue parisienne en zone de guerre urbaine où, loin du regard de l'État incarné de façon fugitive par un fonctionnaire de l'Office Français de Protection des Réfugiés et Apatrides (OFPRA) blasé et une institutrice dubitative, des gangs majoritairement composés de jeunes issus des immigrations d'Afrique subsaharienne et maghrébine se livrent au trafic de stupéfiants en plein jour, armes automatiques au poing et dans le rugissement des V8 de grosses cylindrées de marque allemande. Audiard pose ainsi sur cette société de « sauvageons » le regard d'un autre " sauvage » qui se trouve assigné au rôle « d'autre de l'autre» et rajoute un cran supplémentaire dans l'aliénation de l'autre par l'autre. Dheepan et sa «famille » sont ainsi doublement aliénés : aliénés, car métaphoriquement assignés à résidence en banlieue, ils côtoient d'autres individus aliénés par une République française en panne d'intégration et qui semble ne pas avoir fait le deuil de son passé colonial. En ce sens, le film s'inscrit dans une problématique postcoloniale à plus d'un titre : tout d'abord historiquement, puisqu'il insère le destin d'un tamoul sri-lankais dont la lutte est le résultat de la politique colonialiste des Britanniques sur l'île au cœur de celui des populations françaises d'origine étrangères reléguées dans les «non-lieux » (Augé, 1992) des banlieues ${ }^{7}$. Dheepan est également un film postcolonial grâce au double décentrement effectué, à la fois politique - il s'agit d'un migrant et non plus d'un immigré 8 - et linguistique : en effet la grande majorité des dialogues de ce film français tourné en France est en tamoul. Le film d'Audiard est donc le produit d'une double déterritorialisation, celle de la banlieue parisienne, zone circonscrite par la topographie et la sociologie, puis celle de l'espace mouvant de la diaspora tamoule, entre déplacement, 
emplacement et replacement. Dheepan traduit l'irréductible étrangeté d'un tamoul déraciné face à une banlieue française en déshérence. Au travers de l'épopée d'une famille recomposée par les aléas d'un conflit lointain, il démontre l'impossibilité d'une greffe sociale et culturelle. En revanche, une autre greffe prend, celle du héros tamoul dans une économie cinématographique aux références exclusivement occidentales, comme si la fiction cinématographique et la suspension volontaire de l'incrédulité qui l'accompagne l'emportaient sur les contradictions de la réalité représentée.

Dheepan, au-delà de la critique journalistique ${ }^{9}$ souvent sévère faite à son égard, a le mérite aussi, d'une part, de mettre en lumière une immigration encore mal connue en France, et d'autre part, de montrer comment se fait la maîtrise de la violence, en posant plusieurs questions fondamentales : que veut dire porter la guerre en soi ? Comment renoncer à sa violence en situation de paix ? Comment en faire le deuil ? Qu'est-ce qui change quand on change de place? Dheepan nous donne matière à réfléchir à une triple dynamique de placement : le dé-placement, l'em-placement et le re-placement ${ }^{10}$. Pour Dheepan, devenu ex-combattant du LTTE en France, l'exil représente une période d'individualisation des parcours de vie par rapport à un vécu qui a certainement été marqué par de nombreuses années passées au sein d'une organisation où les membres se sentaient comme dans une famille. Comme le montre Mantovan (2015, p. 91), au sein du LTTE, « la division en castes et les liens de sang font place à la hiérarchie militaire et à la proximité entre camarades ». Comment faire alors pour se re-construire dans ce dé-placement? Comment se dé-faire de ce sujet collectif incarné par la cause ? Ce changement se fait, autour de l'espace et de ses pratiques, à travers les interactions symboliques qui se déroulent par les jeux des corps, les jeux des mots, les jeux des regards que le déplacé porte sur lui-même par rapport aux autres. En d'autres termes, Dheepan montre de quelle manière un déplacé se construit en construisant son monde. Il se confronte au double jeu des mobilités spatiale et sociale. Il se retrouve donc déplacé tant du point de vue des endroits qu'il occupait que des positions sociales qu'il tenait. En questionnant l'habiter de Dheepan, c'est donc cette triple dynamique - dé-placement, em-placement et re-placement - qu'il s'agit de questionner à partir de l'analyse de ses parcours.

\section{Le déplacement : fuir la guerre pour survivre}

6 Dheepan commence par le départ de Sri Lanka du protagoniste principal. Le mystère reste entier sur le type de combattant qu'il a pu être chez les Tigres tamouls. Le premier plan du film est une palmeraie où un combattant se dépouille de son uniforme et le jette sur un bûcher où brûlent des cadavres. Cette séquence d'ouverture nous place dans un décor exotique qui rappelle la palmeraie en feu d'Apocalypse Now (Francis Ford Coppola, 1979) mais, premier décalage, ici le spectacle de la guerre est terminé, l'heure est au dépouillement de l'identité et à la fuite vers d'autres horizons. Le guerrier anonyme achète un passeport au nom d'un mort, « Dheepan », puis reconstitue un ersatz de famille afin de quitter le Sri Lanka : une identité paradoxale, fondée sur ce qui est à la fois une mort symbolique et une renaissance. Précisons néanmoins que les combattants du LTTE ne percevaient pas de salaires durant leur engagement. Au contraire, liés par un engagement contractuel, ils devaient le respecter au risque d'être dans l'obligation de rembourser une partie de leur formation militaire et politique.

7 Pour assurer sa migration vers un pays occidental, Dheepan doit donc se débrouiller comme les autres migrants ${ }^{11}$ - puisque après avoir brûlé son uniforme il perd son statut 
de combattant politique et endosse celui de «Dheepan", une victime civile parmi d'autres - et passer par une agence, le projetant ainsi dans une situation économique précaire. En cela, le film témoigne d'une étape importante, fondatrice, pour le migrant : le déplacement, et plus spécifiquement un déplacement forcé (pour fuir la guerre, des situations de violences, son engagement au sein du mouvement). Ce déplacement forcé est l'expérience de milliers de civils tamouls amenés à trouver des espaces refuges en dehors des zones sous contrôle des LTTE et de l'armée cinghalaise ${ }^{12}$. Pour les civils, il s'agissait de quitter les zones Nord (péninsule de Jaffna et Wanni) et Est (Trincomalee) de l'île. Les premières scènes du film nous donnent à voir cet exode forcé et permet de montrer que l'une des routes migratoires les plus utilisées pour fuir l'île est celle qui relie Talaimannar (à l'extrémité ouest de l'île de Mannar) à Rameswaram en Inde du Sud (Goreau-Ponceaud, 2014b). En effet, si les routes sont multiples, le schéma pour quitter Sri Lanka reste souvent le même: une des premières étapes, non des moindres, est de traverser le détroit de Palk pour se rendre en Inde. La contiguïté géographique, les affinités ethniques et la disponibilité de bateaux font du Tamil Nadu un choix «naturel ». Il faut néanmoins noter qu'à la simplicité des routes essentiellement terrestres des années 1970-1980 (où l'entrée sur le continent européen se faisait via l'Ukraine, la Turquie et Berlin Est) s'oppose la complexité des routes empruntées depuis la chute du Mur de Berlin. Les pays traversés deviennent plus nombreux, allongeant la durée du trajet et le nombre de passages de frontières. Cette complexification des routes, en relation avec l'intensification des contrôles aux frontières, a fait émerger une industrie du passage, qui reposait sur l'organisation hiérarchique mais flexible des LTTE. Malgré ces évolutions, l'entrée sur le territoire européen s'effectue majoritairement par les frontières orientales après avoir combiné différents modes de transport. Ce choix de la transgression des frontières s'appuie sur un réseau de passeurs: les limites entre la simple aide au passage de la frontière et l'exploitation de migrants maintenus dans un état de sujétion, entre le libre choix, la coercition ou la tromperie sont souvent floues, surtout quand il y a dette économique. Cette dangerosité explique en partie que ce déplacement concerne majoritairement des hommes jeunes.

Ce déplacement conduit progressivement Dheepan à quitter son île natale pour l'Inde, pour finalement arriver en France, et plus spécifiquement en région parisienne. Rappelons que, si l'immigration sud-asiatique en France est hétérogène, elle est majoritairement tamoule. Les chercheurs estiment généralement que le nombre de migrants tamouls sri-lankais oscille entre 80000 et 120000 personnes (Goreau-Ponceaud, $2008 \& 2011)^{13}$. Ils seraient ainsi la première communauté d'origine sud-asiatique présente à Paris et en Île-de-France, avec des lieux majeurs d'implantation situés dans les villes de banlieue Nord et Est (petite et grande couronne). Notons que si les Sri Lankais se sont installés en France à partir du début des années 1980, c'est par défaut. Leurs choix se portaient en priorité sur l'Angleterre, en lien avec la phase britannique de colonisation de l'île qui avait privilégié la minorité tamoule, et par la pratique de la langue anglaise qui est restée en héritage. Cependant, du fait du durcissement des lois britanniques sur l'immigration (Immigration Act de 1971 visant à réduire l'immigration en provenance des pays du Commonwealth), les candidats à l'émigration en Angleterre ont dû stopper leur périple en France. C'est ainsi que l'on a assisté, entre les stations de métro La Chapelle et Gare du Nord, entre les $10^{\mathrm{e}}$ et $18^{\mathrm{e}}$ arrondissements de Paris, à la constitution progressive d'une centralité commerciale tamoule (Goreau-Ponceaud, 2008). 


\section{L'emplacement : entre flottement et situations liminaires}

9 La deuxième étape correspond à l'emplacement. Il s'agit de prendre place dans un nouveau contexte national, frappé par une étrangeté singulière. Cette phase, magistralement exprimée par le film, comprend à la fois les défis de l'installation, de l'éducation, du logement, de la demande d'asile, de l'emploi, des réseaux communautaires, de la gestion du traumatisme souvent source de comportements déviants (dans le film, l'alcoolisme a des propriétés apaisantes sur Dheepan et le prévient de comportements guerriers), de la confrontation au pluralisme culturel et religieux et des difficultés liées à une langue et à une culture qu'il ne connaît et ne comprend pas.

Dans cette seconde phase, il est important de préciser que si la constitution progressive d'une centralité commerciale, à La Chapelle, dans le $10^{\mathrm{e}}$ arrondissement de Paris, et l'organisation d'une procession religieuse annuelle ont rendu les Tamouls Sri Lankais de plus en plus visibles, au sein de cette masse d'exilés subsiste une population socialement invisible qui a tendance à ne pas révéler son passé: les anciens combattants du LTTE. Dheepan nous donne à voir cet isolement dont font généralement preuve les anciens combattants du LTTE, qui ne trouvent que peu de soutien auprès des membres de la diaspora, échappant en partie à la solidarité ethnique. Comme a pu le montrer Giacomo Mantovan, les anciens combattants sont généralement privés de reconnaissance sociale en France par les membres de la communauté qui pourtant sont souvent membres d'associations dites nationalistes. En d'autres termes, «leur histoire les contraint à se retirer en un domaine intérieur et à renoncer au rôle public qu'ils exerçaient dans leur existence passée » (Mantovan, 2015, p. 102). Cet exil intérieur vient de l'oubli forcé de la construction de la nation tamoule. Ainsi, cette famille, pour prendre place, ne peut pas compter sur la solidité des chaînes migratoires tamoules, mais doit s'en remettre au dispositif national d'accueil (DNA). Arrivés en France, Dheepan, Yalini et Illayaal, respectivement une jeune femme et une enfant rencontrées dans le camp de déplacés et qui acceptent également d'endosser une nouvelle identité, doivent rapidement faire l'apprentissage d'une langue inconnue, s'inventer une histoire de vie et s'y tenir pour obtenir le statut de réfugié. Il faut d'emblée savoir se faire comprendre, comprendre ce que l'autre veut entendre, savoir le lui dire. À propos des récits de vie des demandeurs d'asile tamouls, Giacomo Mantovan précise qu'en France, «l'aveu constitue la méthode pour produire la vérité que l'OFPRA veut entendre pour accorder le statut » (Mantovan, 2011, p. 45). Ainsi, le filtrage aux frontières par les supposées causes du déplacement est en lui-même une violence faite aux individus. C'est cette famille construite de toutes pièces qui permet à Dheepan de se voir accorder un statut, un logement et un emploi en banlieue parisienne.

11 Les conditions du voyage contribuent également à forger une image marchande de l'asile. En effet, les demandeurs d'asile doivent souvent rémunérer un compatriote pour obtenir la domiciliation nécessaire au dépôt de la demande d'asile, pour effectuer la traduction du récit motivant la demande ou pour se faire accompagner dans une administration inconnue. Ceci s'ajoute au fait que les réseaux d'émigration reposent souvent sur l'endettement. L'entrée des Sri Lankais sur le marché du travail reste donc déterminée par de lourdes obligations financières. Une de celles qui se pose à Dheepan est la constitution d'un réseau actif d'anciens membres du LTTE, témoignant de l'intrication du 
local et du global et du transnationalisme ${ }^{14}$ qui agite la diaspora tamoule. La question de la violence et des gangs tamouls en France est également succinctement évoquée dans le film. Même si Dheepan et sa «famille» semblent être en retrait des réseaux communautaires tamouls, il n'en reste pas moins vrai que le quartier la Chapelle demeure toutefois un point de repère pour eux, notamment parce qu'il y reste une certaine concentration de commerces, de lieux de culte - c'est au temple de Ganesh que se rend la famille dans le film - et d'autres associations qui permettent de tisser des liens par-delà la dispersion.

Dans cette phase d'emplacement, Dheepan se voit confier un métier de gardien, tentant de réparer tout ce qui est à sa portée (ampoules, ascenseur), tout comme de mettre fin à la violence des autres. Cet emploi est en effet hautement symbolique : gardien d'une de ces barres d'immeubles qui sont en France la métonymie de la banlieue, cette fonction incarne un ultime paradoxe. Dheepan l'exilé, le refugié d'un conflit dont personne n'a entendu parler sur sa terre d'accueil, personnifie un certain ordre social représenté par le ménage effectué dans les halls squattés par les dealers et par la distribution du courrier (c'est-à-dire de l'assignation d'identités sociales). Son excentricité - il n'est ni Français de souche, ni d'une des origines coloniales identifiables sur ce territoire urbain - fait de lui un individu assimilé par les petits caïds locaux à l'ordre social. Le révolté devient alors, à leurs yeux, garant symbolique de l'ordre établi. Rapidement Dheepan et sa famille sont amenés à côtoyer la petite pègre du cru. Sa prétendue épouse, Yalini, va se trouver chargée du ménage dans l'appartement où loge un chef de bande, comme s'il fallait indéfiniment tenter de laver et de purifier la violence structurelle ramenée de là-bas ou retrouvée ici. Dheepan nous donne également à voir les différences entre les modes de placement du personnage féminin et masculin. Yalini et Illayaal ont en effet un rapport différent avec leur environnement. Yalini, « l'épouse » de Dheepan, entretient un rapport ambigu avec le fils du vieillard cacochyme qui est le chef de la bande de trafiquants qui règnent sur le quartier et dont elle est l'aide-ménagère. Ces liens qui se tissent restent mystérieux: entre fascination pour la violence et pour cette figure d'Al Capone de troisième zone, et séduction trouble. Yalini et Brahim (Vincent Rottiers) qui joue devant elle le petit dur à la redresse, restent néanmoins sur le seuil d'une relation érotique. Yalini sera le catalyseur de la scène de violence finale - et onirique - où Dheepan se mue en justicier et exécute un par un les « racailles » en montant l'escalier et semble s'en aller délivrer une princesse de conte de fées en haut d'une tour. La question de l'islam salafiste est évoquée brièvement lorsque Dheepan conseille à Yalini de se couvrir la tête afin de se fondre dans l'environnement: ultime décalage ironique où cette question des signes ostentatoires de religion est vue en France par le landernau politique comme un signe évident de non-intégration républicaine. La situation d'Illayaal, «l'enfant» de ce couple, traduit une autre confrontation à la violence symbolique. La jeune fille est en effet scolarisée dans un collège, au sein d'une classe d'adaptation pour " primo-arrivants » et elle se trouve confrontée successivement à l'indifférence d'une enseignante à la moue dubitative (Joséphine de Meaux) face au récit des atrocités de la guerre civile sri-lankaise, puis à la violence physique de ses camarades de classe «maghrébins » et «noirs ». La violence à laquelle sont confrontés les deux personnages du film est différente : Yalini est la spectatrice ambiguë des trafics puis de l'exécution sommaire de Brahim et demeure en marge. Illayaal est en revanche victime d'une violence que l'on peut qualifier de xénophobe de la part de ses camarades issus de l'immigration postcoloniale française. Néanmoins, n'ayant pas été compromises dans le conflit armé sri-lankais, le rapport à la violence est plus éloigné. Alors que Dheepan semble lesté de souvenirs mortifères, Yalini 
et Illayaal paraissent bénéficier d'une mobilité physique plus grande, promesse, peut-être de possible mobilité sociale. Yalini peut frayer avec des criminels tant qu'elle demeure dans son rôle de servante effacée et silencieuse (ce silence et cette incompréhension affichée ne seraient-ils que des stratégies de survie ?). Illayaal quant à elle a accès à l'éducation, c'est-à-dire à l'apprentissage du français et des codes comportementaux qui lui permettent de devenir le truchement du foyer.

Le film joue sur le fait que les personnages échappent à une guerre dans leur pays pour être de nouveau plongés localement dans un autre conflit. De ce dernier toutefois, ils sont simplement spectateurs : ils le regardent par la fenêtre de leur appartement et refusent d'y participer. Cette confrontation à la violence d'ici active le traumatisme du vécu de celle de là-bas. Les scènes où apparaissent de manière progressive un éléphant sont là pour exprimer la résilience de Dheepan. La convocation d'une des figures essentielles de l'hindouisme Shivaïte, Ganesh, permet à Dheepan de prendre acte de l'événement traumatique (les atrocités commises au Sri Lanka, son enrôlement dans le LTTE, sa migration basée sur un mensonge, son détachement du LTTE, la fin de la lutte pour l'Eelam tamoul). Précisons également que même si le LTTE s'est développé en épousant une philosophie néomarxiste laïque, le mouvement s'est néanmoins approprié une imagerie religieuse (autour de Murugan et de Ganesh) ainsi que la dévotion (bhakti) qui imprègne les chansons et les poésies dédiées à la glorification des jeunes combattants qui ont sacrifié leur vie pour la lutte de la libération de l'Eelam tamoul (Goreau-Ponceaud, 2014a). Au final, en Dheepan s'affrontent les gestes de la guerre et ceux de la pacification, comme l'illusoire et pathétique «no-fire zone » (ou zone de sûreté) qu'il trace entre deux barres d'immeubles, afin de se protéger des règlements de compte entre trafiquants de drogue rivaux. "No-fire zone » que Dheepan franchira, conduisant à un massacre au sein de la «cité ». En cela, cette "no-fire zone » nous rappelle les atrocités commises par le gouvernement de Sri Lanka lorsqu'en 2009, il demanda à plus de 400000 civils de s'y rassembler pour les soumettre ensuite à d'intenses bombardements (l'armée prit pour cibles écoles, édifices religieux, camps de réfugiés et hôpitaux), conduisant à la mort de plusieurs dizaines de milliers de civils (majoritairement tués par les forces gouvernementales mais également par les Tigres) ${ }^{15}$.

14 Audiard reprend ainsi dans Dheepan un des tropes du cinéma exilique : le déracinement $\mathrm{du}$ protagoniste qui s'accompagne d'un choc insurmontable entre la culture d'origine et la culture du pays hôte. La question se dédouble puisque la « famille » de réfugiés tamoule se trouve dans l'univers de la banlieue, représentée ici comme une zone de non-lieu où la loi de la République ne s'applique pas, au profit de celle de petits caïds qui font régner la terreur. La déterritorialisation se produit de façon littérale à la fois dans le cadre visuel et dans le cadre sonore du film. L'exil est double et l'étrangeté dialectisée entre celle de la banlieue qui devient paradoxalement pour le spectateur français un lieu identifiable et l'espace intime de la diaspora reconstitué par les dialogues des personnages tamouls et les séquences oniriques de Dheepan, où se rejouent les conflits qui le hantent. Audiard dispose en miroir deux territoires qui se répondent au travers de la famille tamoule et de l'expérience d'ex-Tigre de Dheepan. Pour le cinéaste en effet, zone de guerre sri lankaise et banlieue parisienne livrée à la criminalité sont mises en parallèle sans pour autant que soit livrée une analyse politique au spectateur. Audiard se réfère ainsi à une figure mythique du cinéma américain, celle du vétéran traumatisé de retour dans une vie civile à laquelle il ne peut s'ajuster. 
15 Au personnage d'Ethan Edwards incarné par John Wayne dans The Searchers (John Ford, 1956), au John Rambo de Sylvester Stallone dans First Blood (Ted Kotcheff, 1982) en passant par le Travis Bickle de Robert de Niro dans Taxi Driver (Martin Scorsese, 1976), le cinéma américain a laissé une large place à la figure de cet anti-héros paradoxal. Avatar hollywoodien du Juif errant, le vétéran « désaxé » incarne le retour du refoulé après les traumatismes des différents conflits: Guerre de Corée, Guerre du Viêtnam, autant de conflits lointains créateurs de causes perdues pour des soldats en déphasage incapables de reprendre leur place parmi leurs concitoyens et dont la violence est réveillée par les injustices subies. Dans ces récits virils l'anti-héros vétéran est à la fois le bouc-émissaire et le bourreau nécessaire, le sacrifié et le sacrificateur en marge du monde civilisé, et ainsi son principe rédempteur. Ethan Edwards scalpe l'Indien Scar, le plaçant ainsi du côté obscur et sauvage de la frontière. Travis Bickle, dont l'iroquoise le place également dans le camp des barbares, trucide maquereaux et gangsters dans un bain de sang d'anthologie et John Rambo, le front ceint d'un bandeau évoquant à nouveau sa proximité avec un guerrier amérindien, massacre à la mitrailleuse lourde les rednecks lancés à ses trousses. Dheepan est l'avatar de cette sanglante généalogie où la violence est écartée autant que justifiée par l'enchaînement d'injustices subies. Le héros passe donc par le stade d'un ensauvagement nécessaire, celui d'une ordalie par le sang. Ce retour du refoulé se déroule dans la banlieue française, qui répond avec une série de décalages ironiques au Sri Lanka à l'issue de la guerre civile. La condition de l'exilé est sans cesse déclinée et Audiard met en scène les différentes étapes d'un déracinement.

La langue parlée par Dheepan, Yalini et Illayaal, le tamoul, est l'élément central d'un des aspects du cinéma postcolonial que le critique Hamid Naficy nomme le «cinéma accentué » (accented cinema), c'est-à-dire qui fait partie de l'industrie cinématographique d'un pays hôte (États-Unis, Allemagne, Canada, France, Grande-Bretagne), tout en conservant au travers de ses thématiques, de ses personnages, de son regard décalé, son identité diasporique. En d'autres termes, le cinéma diasporique conserve la syntaxe du cinéma tout en y apportant l'empreinte sonore d'une langue étrangère. Pour Naficy, ce cinéma est une réponse esthétique à l'expérience de l'exil, de la migration ou de la diaspora. La particularité de Dheepan est qu'Audiard mime les caractéristiques du cinéma exilique : en effet, l'une des problématiques centrales de ces films qui «s'expriment avec un accent » (Nacify, 2001) est de dialectiser le rapport entre la langue et le territoire, de souligner la déterritorialisation de l'espace privé du personnage diasporique. Les dialogues en français apparaissent lorsque la «famille» tamoule communique soit avec les représentants d'institutions hexagonales soit avec les habitants de la cité du PréSaint-Gervais. Les échanges en français renvoient sans cesse Dheepan, Yalini et Illayaal à leur étrangeté radicale et "hors-langue», comme si leur expérience était irrémédiablement perdue et littéralement intraduisible. Dans Dheepan, on ne communique pas, on passe de malentendu en malentendu.

17 Le postcolonial dans Dheepan est représenté dans cette assignation d'une population migrante, immigrée ou descendante d'immigrés dans les marges de la topographie du territoire républicain. Comme le remarque Megan MacDonald (2012), la banlieue perpétue l'état d'exception d'une guerre coloniale où les droits individuels sont suspendus et où règne un régime policier qui parque les citoyens de seconde zone dans un soupçon permanent. Pour Dheepan, la seule échappatoire est la violence rédemptrice et un ailleurs sans doute onirique. 


\section{Le replacement : vers une nouvelle migration?}

\section{mauvais niv} évolution professionnelle, ce qui les voue aux petits boulots et à l'économie informelle (vente de jouets à la sauvette et travail d'aide-ménagère dans le film), où ils ont souvent un statut précaire, non salarié et sans protection sociale. Pour beaucoup de ces primoarrivants, l'éducation des enfants est très importante. Dans cette perspective, la nationalité française est envisagée comme un droit qui peut faciliter leur insertion socioprofessionnelle et surtout celle de leurs enfants. Mais pas seulement. L'obtention de la demande d'asile ou de la nationalité française est attendue pour pouvoir quitter la France et s'installer dans un autre pays européen où l'on pense pouvoir vivre mieux. Ce replacement, dans le film d'Audiard, est idéalisé et apparait en total contraste avec l'emplacement, où les scènes sur la banlieue française transpirent d'une portée idéologique politiquement peu correcte. Ce replacement est également surprenant dans le sens où il restaure du conjugal, du familial et ajoute un bébé biologique. L'emplacement selon Audiard est forcément dérégulé, étape transitoire vers le replacement, absolument normé, en Angleterre. Cette phase, très courte dans le film, suggère la mise en regard des « modèles » d'intégration des deux pays, classiquement mis en opposition.

Dheepan est bien un film postcolonial dans le sens où Jacques Audiard nous présente un point de vue décentré vers une marge géopolitique inédite qui renvoie à des problématiques migratoires issues de la décolonisation de l'empire britannique. De plus, ce point de vue « subalterne » (Spivak 1988), du « bas vers le haut » (Young, 2001), fait de Dheepan un film inédit parmi les œuvres cinématographiques hexagonales, où la banlieue est le plus souvent associée au banditisme, comme nous l'avons vu, mais également depuis les années 2000, décrite comme une matrice du djihadisme ${ }^{16}$. Les études postcoloniales peuvent être considérées comme impliquant désormais la plupart des humanités et sciences sociales, non seulement la littérature dont elles sont issues au départ, au tournant des années quatre-vingt, mais également l'anthropologie, l'économie et la géographie. La théorie postcoloniale - objet hybride, aussi vaste que le champ des recherches universitaires lui-même et ancré dans des traditions parfois différentes englobe non seulement ce que l'on peut appeler la « condition postcoloniale » mais aussi les phénomènes de globalisation au niveau d'un système-monde où les réalités nationales se comprennent à l'aune de problématiques transnationales.

\section{BIBLIOGRAPHY}

Althusser L., Balibar E. et al., 1969. Lire le Capital. Paris, Maspero, 688 p.

Appadurai A., 2001. Après le colonialisme. Les conséquences culturelles de la globalisation. Paris, Payot, $322 \mathrm{p}$. 
Augé M., 1992. Non-lieux. Introduction à une anthropologie de la surmodernité. Paris, Le Seuil, 150 p. Boehmer E., 1993. Transfiguring: Colonial Body into Postcolonial Narrative. NOVEL: A Forum on Fiction, vol. 26, n 3, p. 268-277.

Bowen J.R, 2008. Why the French don't like headscarves. Islam, the State, and public space. Princeton and Oxford, Princeton University Press, $304 \mathrm{p}$.

Brubaker R., 2005. The 'diaspora' diaspora. Ethnic and Racial Studies, $\mathrm{n}^{\circ}$ 28, p. 1-19.

Dequirez G., 2011. Nationalisme à longue distance et mobilisation politique en diaspora : le mouvement séparatiste tamoul sri-lankais en France (1983-2009). Thèse de doctorat en sciences politiques, Lille, Université de Lille 2, 384 p.

Fassin D., 2006. Questions sexuelles, questions raciales. In Fassin D. et Fassin E., De la question sociale à la question raciale? Paris, La Découverte, p. 230-248.

Grillo R.D, 2010. An excess of alterity? Debating difference in a multicultural society. In Vertovec S, Anthropology of Migration and Multiculturalism. New-York and London, Routledge, p. 19-38.

Goreau-Ponceaud A. et Gallo E., 2015. L'immigration sud-asiatique : routes migratoires, parcours de santé et intégration. Migrations société, vol. 27, n 161, p. 51-73.

Goreau-Ponceaud A., 2014a. Ganesha Chaturthi and the Sri Lankan Tamil Diaspora in Paris: inventing strategies of visibility and legitimacy in a plural 'mono-cultural' society. In Gallo E., Migration and Religion in Europe. Comparative perspectives on South Asian experiences. Farnham, Ashgate, p. 211-231.

Goreau-Ponceaud A., 2014b. À demeure en exil ? Être refugié tamoul sri lankais au Tamil Nadu. Carnets de géographes, $\mathrm{n}^{\circ} 7$, en ligne. http://www.carnetsdegeographes.org/carnets_recherches/ rech_07_06_Goreau_Ponceaud.php

Goreau-Ponceaud A., 2011. Tamils in France. In Rajan I. et Percot M., Dynamics of Indian Migration. New Delhi, Routledge, p. 64-90.

Goreau-Ponceaud A., 2008. La diaspora tamoule : trajectoires spatio-temporelles et inscriptions territoriales en Île-de-France. Thèse de doctorat (non publiée), 427 p.

Higbee W., Lim S-H, 2010. Concepts of Transnational Cinema: Towards a Critical Transnationalism in Film Studies. Transnational Cinemas, vol. 1, n 1, p. 7-21.

Le Bras H., 2014. L'invention de l'immigré. Paris, L'Aube, 138 p.

Madavan D., 2013. Les minorités tamoules à Colombo, Kuala Lumpur et Singapour : minorités, intégrations socio-spatiales et citadinités. Thèse de doctorat en géographie, Paris, Université Paris-IV Sorbonne, $482 \mathrm{p}$.

Ma Mung E., 2000. La diaspora chinoise. Géographie d'une migration. Paris, Ophrys, 175p.

Mantovan G., 2015. 'Ils étaient les rois'... : l'adieu aux armes d'anciens combattants des Tigres tamouls exilés en France. Migrations société, vol. 27, n 161, p. 89-104.

Mantovan G., 2011. Les récits de vie des demandeurs d'asile tamouls : vers une mémoire collective ? Hommes et migrations, $\mathrm{n}^{\circ} 1291$, p. 39-50.

Meyer E., Madavan D., 2015. Sri Lanka : les séquelles de la guerre. Hérodote, vol. 3, n 158, p. 219-237.

Mucchielli L., 2005. Le scandale des tournantes. Paris: La Découverte, 124 p. 
MacDonald M., 2012. Humanism at the Limit and Post-restante in the Colony: the Prison of the Postcolonial Nation in Jacques Audiard's Un Prophète (2009). International Journal of Francophone Studies, 15, $\mathrm{n}^{\circ} 3 / 4$, p. 561-580.

Naficy H., 2001. An Accented Cinema: Exilic and Diasporic Filmmaking. Princeton and Oxford, Princeton University Press, 392 p.

Rigoulet L., 2015. Dans les coulisses de Dheepan, une aventure sous tension. Telerama, 30 août.

Simon G, 2008. La planète migratoire dans la mondialisation. Paris, Armand Colin, 256 p.

Spivak G, 1988. Can the Subaltern Speak? In Nelson C., Grossberg L., Marxism and the Interpretation of Culture. Urbana and Chicago, University of Illinois Press, p. 271-314.

Wacquant L, 2007. Parias urbains. Ghetto, banlieues, État. Une sociologie comparée de la marginalité sociale. Paris, La Découverte, 331 p.

Young R.J.C., 2001. Postcolonialism: An Historical Introduction. Wiley-Blackwell, Malden and Oxford, $510 \mathrm{p}$.

\section{NOTES}

1. Dans cet article, lorsque Dheepan est écrit en italique, nous faisons référence au film ; sans italique, nous faisons référence au protagoniste éponyme principal du film.

2. Le film de vigilante, du latin vigiles urbani, le veilleur de nuit romain, se traduit par " justicier" ou « auto-justicier». C'est un citoyen qui non seulement se fait justice lui-même mais s'arroge le droit de rétablir l'ordre dans la cité hors de tout cadre légal : à la fois policier, juge et bourreau, il applique une justice expéditive qui rappelle la loi du talion. Ce genre fait florès aux États-Unis, pays du Deuxième amendement autorisant le port et la circulation d'armes, dans les années 1960-1970. Il correspond à un moment charnière dans le cinéma et dans la société américaines : le genre du western est désormais épuisé et le héros de la frontière du Far-West est remplacé par un justicier urbain qui n'hésite pas à faire usage de la violence pour appliquer une loi primaire. L'assouplissement de la censure permet également une représentation plus présente de la violence. En cette période de doutes moraux et de tragédie (assassinat de Kennedy, guerre du Vietnam, désillusion du 'Flower Power'...), de pourrissement social et de tensions raciales persistantes, naît le désir de voir apparaître un Hercule capable de nettoyer les écuries d'Augias de l'Amérique. Le personnage de Batman, personnage de D.C. Comics en est une figure fondatrice, son surnom est en effet The Vigilante. Charles Bronson et Clint Eastwood sont les acteurs les plus marquants ayant incarné des vigilantes au grand écran. Ce genre connaît de nombreuses déclinaisons dans différents avatars de bande dessinée et de héros de série télévisée.

3. Trois critères sont généralement retenus par les géographes (Ma Mung, 2000 ; Simon, 2008) pour définir une diaspora : la multipolarité (dispersion), l'interpolarité (interrelations entre les groupes) et la conscience d'une origine commune. Néanmoins, à l'instar de Brubaker (2005), il s'agit moins de se demander ce qu'est une diaspora (à partir de critères jugés stables et fixes dans le temps) que d'étudier le sens du terme diaspora donné par les individus, les institutions et les organisations (à l'instar du Transnational Government of Tamil Eelam par exemple) qui l'emploient. En ce sens, cette perspective du diasporique qui guide l'analyse d'institutions et d'identités, de liens et de flux dispersés, s'apparente à la notion d'ethnoscapes développée par Appadurai (2001) et souligne le caractère déterritorialisé des communautés imaginées générées par les migrations. 4. Le héros de Taxi Driver (Martin Scorsese, 1976) interprété par Robert de Niro.

5. L'Eelam tamoul est le nom donné par le LTTE (Liberation Tigers of Tamil Eelam) et les associations indépendantistes au territoire qu'ils revendiquent à Sri Lanka, comprenant les 
provinces Nord et Est de l'île. Le tigre est leur emblème. Le mouvement a été fondé en 1972 par Velupillai Prabhakaran, avec comme but affiché la défense des Tamouls par la lutte armée. Pour plus de détails sur le conflit voir Madavan, 2013 et Meyer et Madavan, 2015.

6. Depuis le début des années 2000, les débats nationaux en France comme dans d'autres pays de l'Union Européenne (Pays-Bas, Allemagne et Grande-Bretagne en particulier) ont vu se développer un nouveau thème : celui de la crise, voire de l'échec des modèles d'intégration des migrants ; que ces modèles soient multiculturalistes ou républicains (Bowen, 2008 ; Grillo, 2010 et Goreau-Ponceaud 2014a). La politisation du débat sur la crise et l'échec des modèles ont eu pour résultat de repolitiser très fortement la notion de modèle d'intégration. En France cela a pris la forme d'un discours complexe sur la diversité et la République, particulièrement depuis les «émeutes des banlieues» de 2005. C'est dans ce contexte que lors d'une visite en juin 2015, à la Cité des 4000 de La Courneuve, près de Paris, où un garçon de onze ans avait été tué par balles, que le ministre de l'Intérieur, Nicolas Sarkozy, déclara : «Les voyous vont disparaître, je mettrai les effectifs qu'il faut, mais on nettoiera la Cité des 4000 au Kärcher » (Le Monde, 21 et 22 juin 2015).

7. Marc Augé définit le non-lieu comme l'antithèse du lieu. Le lieu est dans cette perspective un espace habité ou habitable eu égard à ses fonctions identitaires, relationnelles et historiques, alors que le non-lieu en est lui démuni et est par conséquent un espace inhabitable (Augé, 1992). Il y aurait « absence du lieu à lui-même » (Augé, 1992, p. 108). Il peut cependant y avoir une autre lecture pour laquelle le non-lieu n'est pas tant inhabitable qu'habité par un mode de vie autre, d'où son appellation de non-lieu. L'utilisation que nous faisons de ce qualificatif s'inscrit dans cette seconde perspective où la banlieue peut être comprise tantôt comme un espace-écart, un hors-lieu en somme, tantôt comme entre-lieu. Plus généralement, le qualificatif de «banlieue » qui renvoie à un registre binaire, comme s'il existait des « bons » et des « mauvais » quartiers, un centre et une marge, s'inscrit dans un contexte idéologique particulier à la France. Il renvoie à une lecture singulière de la ville en prise directe avec une vulgate politico-médiatique (parfois érigée au rang de doxa) qui se complaît aussi bien dans une approche compassionnelle des quartiers sensibles que dans la dénonciation d'une catégorie spécifique de populations dites « exclues » ou « issues de l'immigration » constitutives d'une catégorie plus large : les «parias urbains » (Wacquant, 2007).

8. Comme le note Hervé Le Bras (2014, p. 22), la crainte de l'immigration, puis de l'invasion, est intimement liée à l'aventure coloniale dont elle inverse les facteurs : la peur de l'invasion s'est imposée comme l'image inversée du désir de conquête et de colonisation. De plus, la constitution progressive d'une « forteresse Europe » s'est accompagnée par une réaffirmation, dans beaucoup d'États européens, d'attitudes racialisantes envers les migrants et les minorités, ces derniers incarnant une altérité indépassable face une identité européenne réifiée et essentialisée (GoreauPonceaud et Gallo, 2015). Dans ce contexte, la distinction idéologique promue dans la sphère publique entre citoyens légitimes et migrants indésirables s'est entrelacée à la force homogénéisante du discours portant sur la panique morale qui serait liée à cette immigration qualifiée de massive. Rappelons, comme le notent Mucchielli (2005) et Fassin (2006), que ces discours relayés par les médias sont soutenus dans les arènes du pouvoir. De façon décisive, quelques études nous invitent à considérer comment, depuis les années 2000, les représentations médiatiques des immigrés (les «Arabes" et les «Noirs" tout particulièrement), dans divers contextes nationaux, se sont moins intéressées à la participation active des étrangers au marché du travail qu'aux effets bouleversants de ces flux irréguliers et non contrôlés sur la culture nationale et son intégrité morale. Dans cette contribution, l'immigré est entendu comme une figure d'outsider qui se construit et que la République a construit à partir d'un rapport négatif à l'identité et à la citoyenneté (comme s'il y avait une dépendance au concept de «fixité » dans la construction idéologique de l'altérité). Cet immigré entretient un lien avec le passé colonial de la France. Dheepan, à l'inverse, se situe en dehors de ce processus d'altérisation issu de contingences 
historiques, médiatiques, politiques, économiques et sociologiques, décrivant des rapports complexes et multiples de domination.

9. Nous pensons à celles publiées par Libération le 25 août 2015, ou encore par les Inrockuptibles le 21 août 2015.

10. Depuis les travaux pionniers de l'École de Chicago, nous savons que la mobilité participe au changement social et à la désorganisation sociale. La mobilité est à la fois un facteur d'enrichissement et de diversification des expériences individuelles, comportant par essence un risque de déstabilisation. Cette triple dynamique de placement possède donc une double lecture : à la fois sociale et spatiale.

11. Notons que la différenciation entre migrants et réfugiés est devenue plus floue, de même que les catégorisations entre pays de départ, d'accueil et de transit. Le migrant lui-même, au cours de sa vie, peut avoir successivement une multiplicité de statuts: touriste, demandeur d'asile, clandestin, réfugié. C'est ce que nous donne à voir Dheepan.

12. Le Sri Lanka a été le théâtre, de 1983 à 2009, d'une guerre civile meurtrière qui a eu de larges répercussions dans les logiques migratoires des Sri Lankais. Terre d'immigration depuis les débuts de son histoire, le Sri Lanka est devenue progressivement une terre d'émigration massive. La mise en place de lois discriminantes (Sinhala only Act de 1956 en particulier) a lancé sur les routes de l'exil, de manière sporadique dans un premier temps, des élites anglophones vers les pays du Commonwealth. Néanmoins, après les pogroms anti-tamouls de juillet 1983, correspondant également à la montée en puissance du mouvement du LTTE, les flux de réfugiés et de demandeurs d'asile en direction des pays du Nord et d'Asie (Malaisie et Inde en particulier) sont devenus significatifs. Notons que dans la société sri lankaise se juxtaposent des communautés qui diffèrent par la langue, la religion et les structures sociales. D'origine indoaryenne (du nord de l'Inde) et à grande majorité bouddhiste, la communauté cinghalaise est la population majoritaire dans le pays. La population tamoule, d'origine dravidienne (sud de l'Inde), est scindée en deux ensembles : les Tamouls "autochtones" et les Tamouls des plantations, qui ont un statut et des intérêts très différents. Les Tamouls "autochtones", présents bien avant la colonisation aux côtés des Cinghalais, ont le sentiment d'être, tout comme ces derniers, des "enfants du sol" et non des immigrés. Les Tamouls des plantations, arrivés au XIX ${ }^{\mathrm{e}}$ siècle, ont été utilisés, sous la domination britannique, comme main-d'œuvre dans les plantations du centre du pays, enclavées dans des régions à forte majorité cinghalaise.

13. Ces chiffres ne tiennent pas compte de la diversité des statuts (entre les demandeurs d'asile, les déboutés du droit d'asile, les réfugiés, les naturalisés) et comprennent les descendants des migrants.

14. Notons qu'en termes de migrations transnationales et de relations internationales, la dimension extra-insulaire du conflit sri-lankais est significative. On évalue l'importance des diasporas, principalement tamoules, à plus d'un million de personnes (si l'on compte les descendants des migrants). Ces réseaux transnationaux défient la souveraineté de l'État-nation par l'importance des liens entre les différents pôles de la diaspora, par-delà les frontières, nourrissant un nationalisme à longue distance (Dequirez, 2011).

15. Comme le mentionnent Eric Meyer et Delon Madan (2015, p. 224) «le décompte des victimes est l'objet de vives controverses qui opposent le gouvernement de Colombo aux ONG ».

16. Les meilleurs exemples sont certainement Désintégration (2001) de Philippe Faucon et Made in France (2014) de Nicolas Boukhrief. Pour reprendre une expression qui fait florès, «l'islam des banlieues » a désormais remplacé la banlieue des sauvageons dans l'imaginaire apocalyptique des médias et de la classe politique française qui, faisant fi de toute perspective historique, ne lisent ces phénomènes que d'un point vue exclusivement "franco-français », oubliant ainsi la dimension postcoloniale du phénomène islamique. Malgré son titre extrêmement racoleur, le dernier ouvrage de Gilles Kepel, Terreur dans l'Hexagone, genèse du djihadisme français (Paris, 
Gallimard, 2016), replace les questions d'échec de l'intégration des populations issues des colonies françaises dans une perspective historique.

\section{ABSTRACTS}

Dheepan questions a contemporary trope, that of a migrant from a state torn apart by an internal war that raged from July 1983 to May 2009 between the army of Sri-Lanka and the separatist Tamil Tigers struggling for the liberation of Tamil Eelam. The migrant hero is placed in another hostile environment, that of the inner suburbs of Paris where other problems confront him. Three phases of diaspora are described: displacement in order to survive, replacement in a urban liminal area, and finally location in an idyllic territory.

Dheepan interroge un trope contemporain, celui du migrant issu d'un État déchiré par une guerre intestine - celle opposant de juillet 1983 à mai 2009 l'armée sri-lankaise aux militants séparatistes tamouls des Tigres de libération de l'Eelam Tamoul (LTTE) - en le plaçant dans la perspective de la banlieue parisienne, univers urbain où se déploie une autre problématique. Ainsi se trouvent décrits les trois moments de la migration du diasporique : le déplacement pour survivre, l'emplacement dans une marge urbaine et enfin le replacement dans un territoire idyllique.

\section{INDEX}

Keywords: Audiard, cinema, diaspora, French inner cities, immigration, Tamils, Sri-Lankan, postcolonial.

Mots-clés: Audiard, banlieue, cinéma, diaspora, France, immigration, Tamouls, Sri-Lankais, postcolonial.

\section{AUTHORS}

\section{ANTHONY GOREAU-PONCEAUD}

Anthony Goreau-Ponceaud, anthonygoreau@yahoo.fr, est Maître de conférences à l'Université de Bordeaux et membre de l'UMR 5115 LAM « Sciences Po Bordeaux ». Il a publié récemment :

- Goreau-Ponceaud A. et Gallo E. (dir.), 2015. L'immigration sud-asiatique en questions. Migrations et société, vol. 27, n 161, p. 49-220.

- Goreau-Ponceaud A. et Veyret P. (dir.), 2015. Indian values: Diaspora and Womanhood. DESI, $\mathrm{n}$

- 3, $200 \mathrm{p}$.

- Goreau-Ponceaud A., 2014. De Ceylan Quarter à Keezhputhupattu : lieux de l'exil sri-lankais en Inde. Les Cahiers d'outre-mer, vol. 67, $\mathrm{n}^{\circ}$ 267, p. 261-284.

\section{PAUL VEYRET}

Paul Veyret, Paul.Veyret@u-bordeaux-montaigne.fr est Maître de conférences en études anglophones et membre de l'EA 4196 Climas à l'Université Bordeaux Montaigne. Il a publié 
récemment :

- Goreau-Ponceaud A. et Veyret P. (dir.), 2015. Indian Values: Diaspora and Womanhood. DESI, $\mathrm{n}$ 3, 200 p.

- Veyret P., 2015. Deepa Mehta's Troubled Water: Engaging Indian Values. DESI, n³, p. 125-139.

- Veyret P., 2015. Les eaux silencieuses et le «traumatisme » de la Partition. A paraître. 